\title{
Time-gating processes in intra-cavity mode-locking devices like saturable absorbers and Kerr cells
}

\author{
Narasimha S. Prasad \\ NASA Langley Research Center, 5. N. Dryden St., MS 468, Hampton, VA 23681 \\ narasimha.s.prasad@nasa.gov
}

and

Chandrasekhar Roychoudhuri

Department of Physics, University of Connecticut, Storrs, CT 06269

chandra@phys.uconn.edu

\begin{abstract}
Photons are non-interacting entities. Light beams do not interfere by themselves. Light beams constituting different laser modes (frequencies) are not capable of re-arranging their energies from extended timedomain to ultra-short time-domain by themselves without the aid of light-matter interactions with suitable intra-cavity devices. In this paper we will discuss the time-gating properties of intra-cavity "mode-locking" devices that actually help generate a regular train of high energy wave packets.
\end{abstract}

Key words: Photons, light-matter interaction, Mode-locking, Kerr devices

\section{CONTRADICTIONS IN MODE LOCK THEORY \& RESULTS}

The purpose of this paper is to draw attention to the community, involved in promoting and developing the technologies of ultra short pulse generation using various kinds of laser cavities incorporating "mode locking" devices, that we need a new mode of modeling and analysis that can represent actual physical processes behind the short pulse generation [1,2]. Analysis of the output spectrum (carrier frequency distribution) indicates that the physical process behind short pulse generation is not "mode locking". It is simply time-gating or Q-switching, although down-selection of in-phase spontaneous and stimulated emission is of tremendous help in initiating and sustaining the ultra short pulse generation. However, the dominant role is played by the time-gating properties of the intra-cavity devices.

Consider the optical spectrum from a "mode-locked" He-Ne laser shown in Fig.1. According to mode lock theory, the nano second pulse train in Fig.1a implies that output optical spectrum should display one single mean frequency given by the Fourier summation of the periodic longitudinal modes.

$$
E_{\text {total }}\left(v_{0}, t\right)=\sum_{n=0}^{N-1} e^{i 2 \pi\left(v_{0}+n \delta v\right) t+i \phi_{c}} \approx N \frac{\sin N \pi t / \tau}{\sin \pi t / \tau} e^{i 2 \pi v_{0} t+i \phi_{c}} \equiv N a(t) e^{i 2 \pi v_{0} t+i \phi_{c}}
$$

However, the measured optical spectrum in Fig.1b shows the presence of all the longitudinal modes that an inhomogeneously broadened gain medium can sustain. Heterodyne measurement of the width of the individual optical spectral lines shows ultra narrowness, $10 \mathrm{KHz}$, indicating that the longitudinal modes are highly phase locked ("coherent"). These results imply that for a "mode-locked" He-Ne laser either the modes really do not sum to a mean frequency, as implied by a simplified mode-lock Eq.1, or the generation of short pulses is really due to some Q-switching (time-gating) process, rather than "mode-locking". 


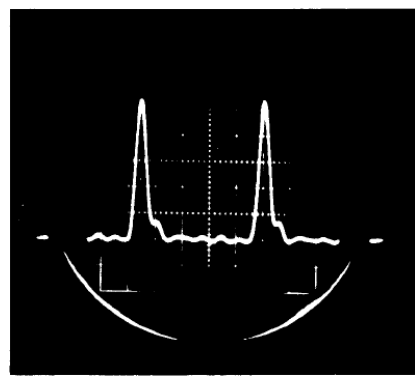

a)

Mode-locked pulses train

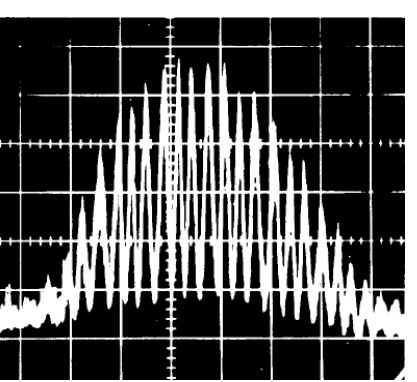

(b)

Longitudinal mode spectrum $10 \mathrm{kHz}$ narrow intrinsic line width

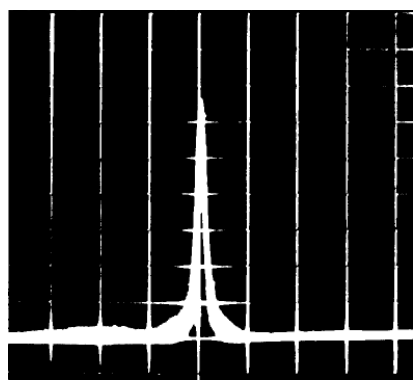

(c)

Figure 2. Experimental data from a mode-locked He-Ne laser showing intensity vs. time for a mode-locked pulse train shown in (a); the longitudinal mode spectrum is shown in (b), and the high resolution line width, $10 \mathrm{kHz}$, of one individual laser mode is shown in (c) [3].

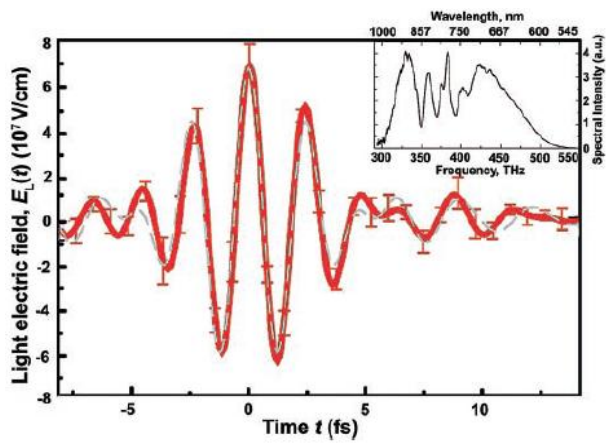

Figure 2. Demonstration of the existence of a single carrier frequency in a 4.5 fs pulse by directly measuring the harmonically undulating E-vector undulation [4].

Consider now the measured E-vector undulation in a femto second pulse (Fig.2) derived from a highly stabilized "mode locked" laser using homogeneously broadened T-Sapphire laser. It is clearly a single carrier frequency. Now, one can argue in favor of traditional mode lock theory and Eq.1 as if all the cavity longitudinal modes have really summed up to produce this single carrier frequency [5].

Unfortunately, this argument demands the assumption that a perfectly homogeneously broadened spectral characteristic of Titanium atoms inside a homogeneous Sapphire crystal becomes inhomogeneous due to remotely imposed time-gating characteristics by the intra-cavity "mode-locking" device. The other alternative is to seek refuse under the time-frequency Fourier theorem (TF-FT). Slightest periodic modulation of the intensity in the cavity will generate Fourier frequencies, which will automatically coincide with the cavity longitudinal modes and promote their oscillation in phase and hence the carrier frequency of the output pulses will have the characteristics as has been measured (Fig.2). While the TF-FT solves the case for the T-Sapphire laser, the case for He-Ne laser remains unsolved. But riding on the shoulder of the TF-FT has another serious problem. It implies that TF-FT correctly models some inherent physical processes in nature experienced by light beams corresponding to different frequencies; they regroup their energies into temporal pulses. If this were true, then we should have declared TF-FT as a principle of nature. It is fortunate that we have not done so. According to quantum mechanics, the photons are non-interacting Bosons. While classical physics has supported the concept that light interferes, or EM waves sum themselves to regroup their energy in space and time. In reality, it is an untenable assumption that can be appreciated by analyzing our daily observations with any kinds of propagating waves. We will 
justify the NIW-principle (Non-Interference of Waves) as a generalized behavior of all kinds of self perpetuating propagating waves in the next section.

\section{THE NIW-PRINCIPLE (NON-INTERFERENCE OF WAVES)}

If we pay careful attention to propagating water waves, we will notice the NIW-principle in action. All coherent wave groups propagate through each other unperturbed, even though within the volume of real physical superposition, we can see the resultant amplitudes are affected by local superposition effects. Same is true for propagating sound waves. Otherwise, we would never been able to maintain any conversations during any parties we attend $[6,7]$.
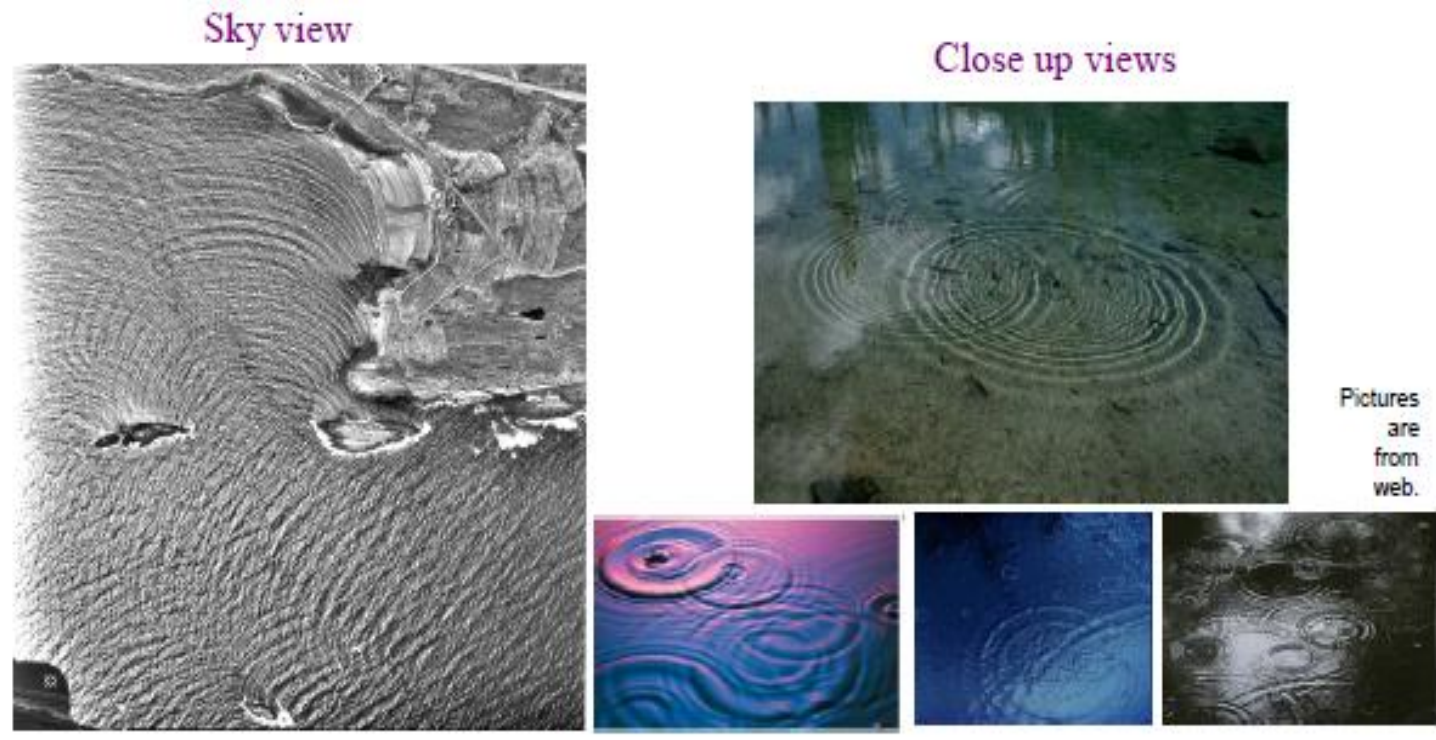

Figure 3. Perpetually propagating surface water waves underscores the NIW-principle in daily life. While the resultant displacement patterns of the wave-sustaining-medium change locally due to superposition of multiple waves, each coherent wave group passes through each other unperturbed, without interfering with each other, as if they have never experienced each other's presence [Pictures gathered from the web].

The situation for light beams is more subtle since the medium through which light propagates, is as yet, invisible to us. However, we can still recognize the NIW-principle by simply being careful observant (see Fig3). Crossing or co-propagating light beams carry on with their original information unperturbed by each other's presence. Otherwise, our daily visual world, the technological world of WDM communication and the scientific study of distant stars (cosmology) would have been impossible. 

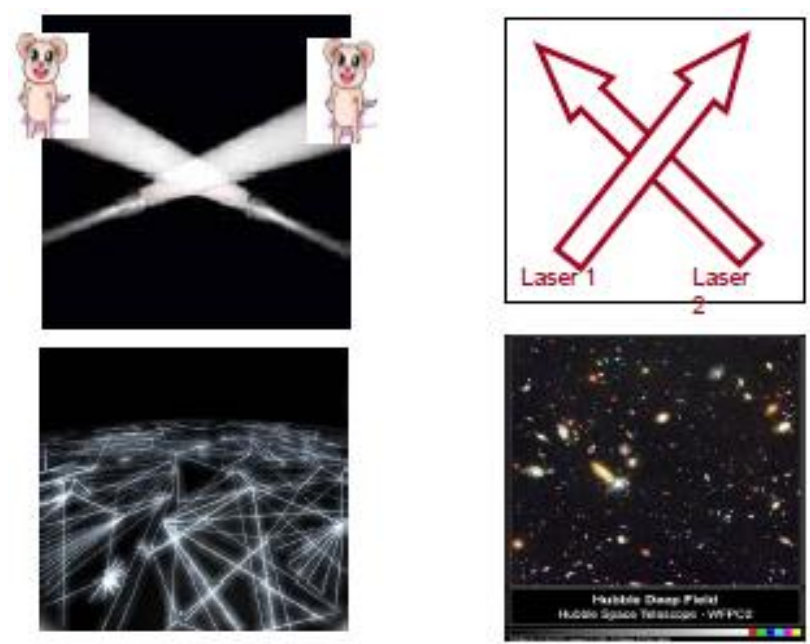

Figure 3. The NIW-principle is obvious from simple observations. If light interacted (interfered) with light, then: (i) images in the visual world would have been full of spatial and temporal scintillations (speckles); (ii) energy distribution of laser beams would have been different before and after crossing; (iii) WDM internet data would have been destroyed by temporal interference (heterodyne effect) and (iv) the expanding universe, indicated by Doppler shift, would have been very noisy \{pictures collected and synthesized from the web].

Thus, the NIW-principle forbids our basic assumption behind mode locking. We cannot simply sum amplitudes of periodic frequency to obtain re-grouped energy distribution in time. Propagating waves do not sum themselves.

$$
\begin{aligned}
E_{\text {total }}\left(\nu_{0}, t\right) & \neq \sum_{n=0}^{N-1} e^{i 2 \pi\left(v_{0}+n \delta v\right) t+i \phi_{c}} \approx \frac{\sin N \pi \delta v t}{\sin \pi \delta v t} e^{i 2 \pi v_{0} t+i \phi_{c}} \\
& \neq N a(t) e^{i 2 \pi v_{0} t+i \phi_{c}}
\end{aligned}
$$

Thus, the TF-FT is also a non-physical model since it sums propagating amplitudes:

$$
\begin{gathered}
a(t)=\int_{0}^{\infty} \tilde{a}(f) \exp [-i 2 \pi f t] d f \\
\tilde{a}(f)=\int_{0}^{\infty} a(t) \exp [i 2 \pi f t] d t
\end{gathered}
$$

In spite of mathematical congruency of the theorem and its applicability in wide ranges of physical phenomenon, we should remain vigilant as to where the theorem is applied appropriately. We have shown elsewhere how we have mistakenly interpreted broadening of spectral fringes of pulsed light due to timediffraction as new optical spectrum due to some fortunate coincidences with the TF-FT theorem, Parseval's theorem on the conservation of energy and the time-integrated spectrum that we normally record [8-10].

However, electrical engineers should be happy to know that classical LCR-circuits can sum the radio frequency induced oscillatory currents. Here Fourier theorem works because mathematics matches the physical process:

EM field: $E\left(t, v_{1}\right)=a \cos 2 \pi v_{1} t$;

Current: $I_{L C R}\left(t, v_{1}\right)=\eta a \cos \left(2 \pi v_{1} t+\varphi\right)$;

Voltage: $V_{L C R}\left(t, v_{1}\right)=\eta a \cos 2 \pi v_{1} t$

Power: $I_{L C R} V_{L C R}=\eta^{2} a^{2} \cos 2 \pi v_{1} t \cos \left(2 \pi v_{1} t+\varphi\right)$ 


$$
\begin{aligned}
& E_{1,2}\left(t, v_{1,2}\right)=a \cos 2 \pi v_{1,2} t \\
& I_{L C R}\left(t, v_{1}, v_{2}\right)=\eta a\left[\cos 2 \pi v_{1} t+\cos 2 \pi v_{2} t\right] \\
& \quad=2 \eta a \cos 2 \pi \frac{v_{2}+v_{1}}{2} t \cos 2 \pi \frac{v_{2}-v_{1}}{2} t
\end{aligned}
$$

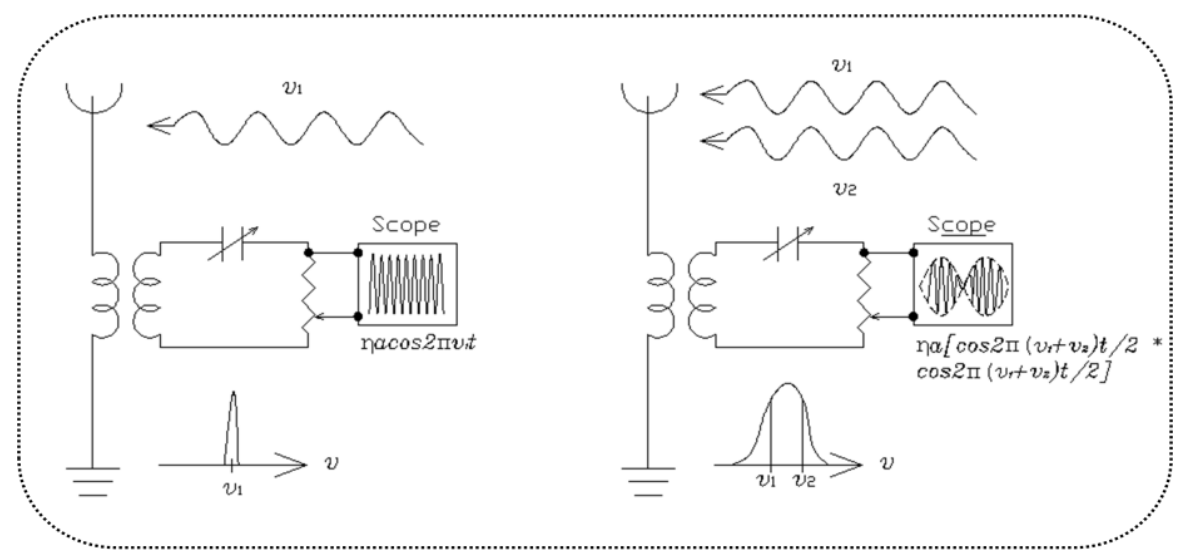

Figure 4. Fourier theorem correctly models undulatory "swishing" of a bundle of conduction electron in an LCR-circuit when it is pushed by undulatory potential difference induced by different radio frequencies $[\mathrm{x}]$.

The circuit current due to two radio frequencies is a sum of two sinusoids emulating the incident fields. Thus the current is amenable to Fourier analysis without any arbitrary assumptions. If we normalize this Eq. to eliminate the constant $\eta a$, we can easily confuse ourselves that the two radio waves have interfered with each other (summed themselves). The linear summation of the sinusoids in the "AC" current (resultant "swishing" of the "free" conduction electrons) has been carried out by the LCR circuit response, not the propagating radio waves, even though the energy transfer is quadratic. An oscilloscope can measure the "mean of the sum frequencies" and the "mean of the difference frequencies", as shown in Eq.6.

For optical detectors, the energy transfer is also quadratic, but the frequency information is lost during the quantum mechanical "release" process. The photo electric current is produced when the bound quantized electrons are transferred "one way" to the "free" conduction band, to be extracted by applying some external voltage! Thus, a photo detector cannot produce the "mean of the sum frequencies" and the "mean of the difference frequencies", as shown in Eq.6. A photo detector can only produce an undulatory "DC" current including the "difference" or the beat frequency, because the energy transfer process is the square modulus of the complex amplitudes:

$$
\begin{aligned}
I(t) & =\left|{ }^{(1)} \chi a e^{-i 2 \pi v_{1} t}+{ }^{(1)} \chi a e^{-i 2 \pi v_{2} t}\right|^{2} \\
& =2^{(1)} \chi^{2} a^{2}\left[1+\cos 2 \pi\left(v_{1}-v_{2}\right) t\right] \\
& =2^{(1)} \chi^{2} a^{2}[\mathrm{DC} \text { for slow detector circuit }]
\end{aligned}
$$

Here ${ }^{(1)} \chi$ is the quantum mechanical polarizability of the detecting molecules and $\left(v_{1}-v_{2}\right)$ is the "difference" or the beat frequency. Thus, optical photo detectors, by virtue of their quantum mechanical transformation process, cannot simulate Fourier summation of different frequencies. Note that by adding extra circuit time-constant external to the photo detector, one can average out the undulatory "DC" current into a steady "DC" current. Thus, optical superposition effect really represents the characteristics of the detecting system, although the stimulations and the energy are provided by the superposed fields. 


\section{KERR-LENS IS A CAVITY-SPATIAL-MODE SELECTOR!}

We have demonstrated in the last two sections that summation of the longitudinal modes (mode locking) is not the real physical process behind ultra short pulse generation by laser cavities. We believe that the fundamental process is Q-switching or time-gating induced by the "mode locker" (saturable absorber, Kerr cell etc., with or without temporal modulation). Of course, the spontaneous selection of inphase emissions by the "mode locker" enhances the saturable absorption or nonlinear lensing of the Kerr cell, which are then selected by the "opened" cavity for further coherent amplification. In fact, the Kerr lens as a spatial TEM $_{00}$ mode selector (Fig.5) is one of the best case to start modeling the evolution of short pulse as time-diffraction, rather than "traditional "mode locking". One should start with the first principle. First model the time diffraction of spontaneously emitted "semi exponential" photon wave packets with their own unique carrier frequency (see model in 14) starting with the spontaneous emissions. In time diffraction, one propagates the carrier frequency of the pulse, not the Fourier frequencies of the temporal envelope [15]. Then, incorporate the temporal evolution of the nonlinear focusing effect in the Kerr cell as interaction between the bulk Kerr medium and multiple superposed photon wave packets until one obtains a steady sate time-gating effect out of the Kerr cell.

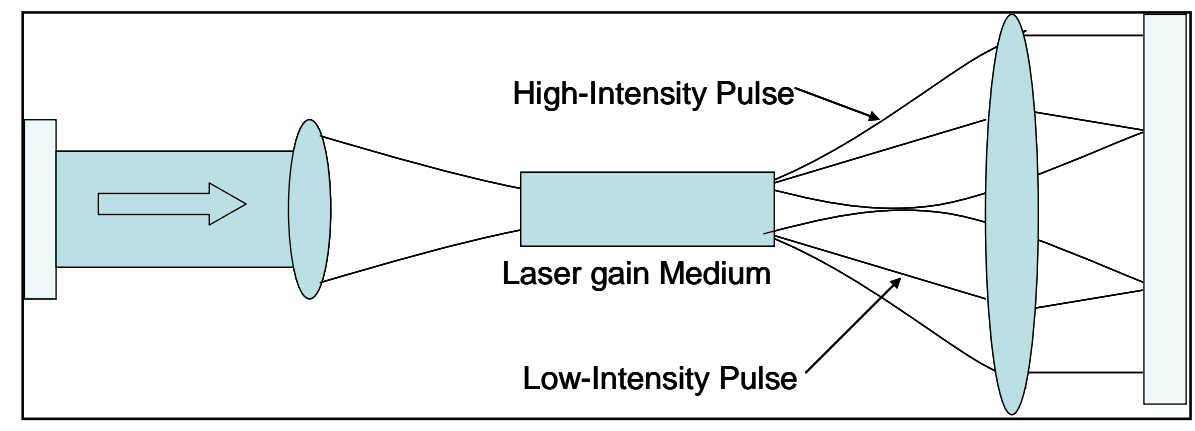

Figure 5.Kerr Lens mode-locking scheme [16, 17]

\section{SUMMARY}

1. The NIW-Principle - Non-Interference of Waves: Photons or propagating wave packets (or their complex amplitudes) pass through each other unperturbed. Waves do not interact to redistribute their energy without the aid of some suitable material media.

2. Longitudinal modes in a cavity, in or out of phase, cannot re-constitute their energy to create temporal pulses. This is dictated by the NIW-principle.

3. All pulsed lasers are Q-switched of some form aided by the intra-cavity time-gating material devices. Classical non-linear bulk property of Kerr medium provides a faster switch than saturable absorbers, driven by quantum mechanical band transition .

4. The use of the time-frequency Fourier theorem (TF-FT) to model "mode-locking" diverts us from discovering the real physical time-gating processes. By virtue of the NIW-principle, TFFT cannot be a principle of nature. It is a limited tool only

5. Temporal evolution of cavity spatial mode and phase coherence of stimulated emissions in time are the real physical parameters for modeling short pulse lasers. Attempts to distinguish between the physical processes behind "phase-locked" Q-switching of gain media with homogeneous and inhomogeneous spectral broadening of their gain band-widths.

6. The NIW-principle is already built into the accepted classical and quantum theories. We just failed to discover it for three hundred years! Huygens' principle works only because the complex 
amplitudes in any forward plane can be computed from those in any previous plane. Second quantization was forced to accept photons as non-interacting Bosons!

7. Physics must to be revitalized to model invisible physical processes behind all interactions. We have been modeling just the observables, instead of the interaction processes that give rise to the observables (measurables)!

\section{CONCLUSIONS}

1. All pulsed lasers are Q-switched devices with some time-gating technique(s) built into the cavity to achieve temporal gain synchronization.

2. Pulsed laser analysis should focus on modeling temporal evolution of cavity spatial-mode, dictated by the properties of gain medium.

3. The NIW-principle supersedes other modeling approaches in spite of their apparent successes.

\section{REFERENCES}

1. C. Roychoudhuri and N. Prasad "Various ambiguities in re-constructing laser pulse parameters", proceedings of the October, 2006 IEEE-LEOS Annual Conference, Montreal, Canada; Invited Talk.

2. C. Roychoudhuri and N. Prasad, "Light-matter interaction processes behind intra-cavity mode locking devices"; SPIE Conf. Proceedings Vol.7193-67 (2009).

3. L. B. Allen, R. R. Rice and R. F. Mathews, "Two cavity mode-locking of a He-Ne laser", Appl. Phys. Lett., Vol.15, pp.416-18 (1969).

4. Goulielmakis et. al., "Direct Measurement of Light Waves"; see Ch.27 in The Nature of Light: What Is a Photon? Eds. C. Roychoudhuri, A. F. Kracklauer \& Kathy Creath; CRC Press (2008); see also Science Vol. 305, 27Aug. 2004.

5. A. Siegman, Lasers, University Science Books (1986).

6. C. Roychoudhuri, "The NIW (Non-Interference of Waves) principle removes wave-particle-duality and "spooky" conjectures." Foundations of Physics (2010); accepted for publication.

7. C. Roychoudhuri, "Inevitable incompleteness of all theories: an epistemology to continuously refine human logics towards cosmic logics"; Ch.6 in The Nature of Light: What is a Photon? by C. Roychoudhuri, A. F. Kracklauer \& Kathy Creath; CRC/Taylor \& Francis (2008).

8. C. Roychoudhuri, "Propagating Fourier frequencies vs. carrier frequency of a pulse through spectrometers and other media"; Proc. SPIE Vol.5531, 450-461(2004).

9. C. Roychoudhuri and M. Tayahi, Intern. J. of Microwave and Optics Tech., July 2006; "Spectral SuperResolution by Understanding Superposition Principle \& Detection Processes", manuscript ID\# IJMOT2006-5-46: http://www.ijmot.com/papers/membercheck.asp?id=IJMOT-2006-5-46.

10. C. Roychoudhuri, Phys. Essays 19 (3), September 2006; "Locality of superposition principle is dictated by detection processes".

11. C. Roychoudhuri and P. Poulos, SPIE. Proc. Vol.6664 (2007); "Can we get any better information about the nature of light by comparing radio and light wave detection processes?"

12. [C. Roychoudhuri, "The NIW (Non-Interference of Waves) principle removes wave-particle-duality and "spooky" conjectures." Foundations of Physics (2010); accepted for publication.

13. C. Roychoudhuri, "Inevitable incompleteness of all theories: an epistemology to continuously refine human logics towards cosmic logics"; Ch.6 in The Nature of Light: What is a Photon? by C. Roychoudhuri, A. F. Kracklauer \& Kathy Creath; CRC/Taylor \& Francis (2008).

14. C. Roychoudhuri and N. Tirfessa, Proc. SPIE Vol.6372-29 (2006), "Do we count indivisible photons or discrete quantum events experienced by detectors?".

15. C. Roychoudhuri, "Propagating Fourier frequencies vs. carrier frequency of a pulse through spectrometers and other media"; Proc. SPIE Vol.5531, 450-461(2004).

16. See Kerr effect discussion and listed references in http://en.wikipedia.org/wiki/kerr_effect

17. See Kerr lens based mode-locking discussions in http://www.physics.gatech.edu/frog/lectures/UltrafastOptics/lectures/UFO18Mode-lockingtheory.ppt and Hermann Haus, "Short pulse generation," in Compact Sources of Ultrashort Pulses, Irl N. Duling, ed. (Cambridge University Press, 1995). 